\title{
MINIATURE WIRELESS RESONANT ROTARY MOTOR ACTUATED BY LITHOGRAPHICALLY MICROMACHINED MAGNETOELASTIC FOIL
}

\author{
J. Tang, S.R. Green, Y.B. Gianchandani \\ Center for Wireless Integrated MicroSensing and Systems (WIMS ${ }^{2}$ ), University of Michigan, \\ Ann Arbor, Michigan, USA
}

\begin{abstract}
This paper presents a miniature rotary motor that is driven by wireless power transfer. This resonant motor is actuated by a $\varnothing 8$ $\mathrm{mm}$ magnetoelastic stator lithographically micromachined from Metglas $^{\mathrm{TM}} 2826 \mathrm{MB}$ foil. The prototype motor operates at a resonant frequency of $11.35 \mathrm{kHz}$ while a 3 Oe DC and a 2 Oe amplitude AC magnetic field are applied. The measured rotation speed, calculated stall torque, and output power are $44 \mathrm{rpm}, 2$ $\mathrm{nN} \cdot \mathrm{m}$ and $9 \mathrm{nW}$, respectively. The angular resolution is $\sim 23$ millidegree. Based on simulation results and the experimentally measured performance of stator prototypes, simulation parameters are derived and used in predicting the performance of a siliconmetglas stator. A significant improvement in rotation speed, resolution, and required power is predicted.
\end{abstract}

\section{INTRODUCTION}

Chip-scale rotary motors have been of interest since the 1980s. Potential applications include actuation of optical elements in microsystems for communication or microanalytical applications, or for the in-situ calibration of inertial sensors particularly rotational rate and rate-integrating sensors. Another possible application for a wirelessly-powered miniature motor is to provide propulsive force for microbots in cavities and ducts. Motor actuation methods have included electrostatic, piezoelectric, electromagnetic, and thermal [1-4]. Electrostatic motors are considered as one of the milestones in MEMS history and are simple to integrate with IC processes, but packaging requirements, output torque and payload capacity limit applicability. Piezoelectric motors have high output torque. Electromagnetic motors are attractive but present challenges for the integration of coils. In this paper, another option for chip-scale rotary motor, magnetoelastic actuation is investigated.

Magnetoelastic coupling represents the interaction between the material conditions of strain, stress and magnetization. Magnetoelastic materials exhibit strains under an external magnetic field due to the field-directed rotation and alignment of tightly-coupled, elongated structural and magnetic domains in the materials. The strain induces stress in the material which, in turn, alters the magnetization. When excited with an oscillatory field, these two simultaneously existing effects make magnetoelastic materials attractive for wireless resonant sensing. Various magnetoelastic sensing systems have been studied for pressure, temperature, liquid viscosity and density, fluid flow rate, $\mathrm{pH}$, glucose, Young's modulus, stent occlusion, etc. [5]. Utilizing magnetoelastic material for actuation of a miniature and microscale remains an open challenge. Magnetoelastic actuators can operate by mechanically resonating in response to an alternating magnetic field. The resonant motion is used to drive movement of other parts of the system. The use of magnetoelastic transduction enables wireless actuation, which simplifies device architecture and allows for remote operation. The transduction method also exhibits high payload carrying capacity as well as noise immunity due to resonant operation.

The approach followed in this effort is similar to ultrasonic rotary motors, which are typically actuated by piezoelectric or electrostatic means [6-8] and operate by generating a vibratory wave in the stator. In this case, the vibratory micro-motion is a standing flexural resonant wave and is generated magnetoelastically. The resulting vibratory shape of the stator has antinodes at which maximal out-of-plane deflection occurs. Teeth are located on the rotor such that they are slightly offset from the antinodes, resulting in a contact force with a tangential component that causes the rotor to rotate (Fig. 1). A rotor, which can contain other microsystem components, is then stacked on to the stator and rotates on the motor hub. The prototype motor presented in this paper is $8 \mathrm{~mm}$ in diameter and is able to carry loads up to $9 \mathrm{mg}$; a magnetoelastic material Metglas ${ }^{\mathrm{TM}} 2826 \mathrm{MB}$ is utilized to actuate a chip-scale rotary motor.

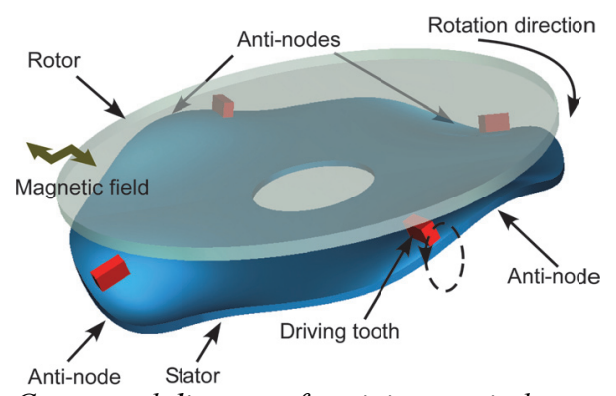

Figure 1: Conceptual diagram of a miniature wireless magnetoelastic resonant rotary motor.

\section{DESIGN AND MODELING}

A prototype megnetoelastic wireless resonant miniature motor is assembled by stacking a magnetoleastic stator and two stainless steel bases (Fig. 2). The stacked architecture is modular, which significantly simplifies the fabrication process. Each layer can be easily fabricated utilizing photochemical machining (PCM) process, as described in the fabrication section. Alignment pins ensure reasonable assembly accuracy for a stator with a diameter of $8 \mathrm{~mm}$. Two stainless steel bases provide a recess allowing stator vibration and initial positions for alignment pins and a hub. The ring-shaped stator is suspended with four crab-leg springs, which are stiff in the rotational direction but flexible in the out-ofplane direction - preventing stator rotation during rotor actuation while allowing large vertical deformation.

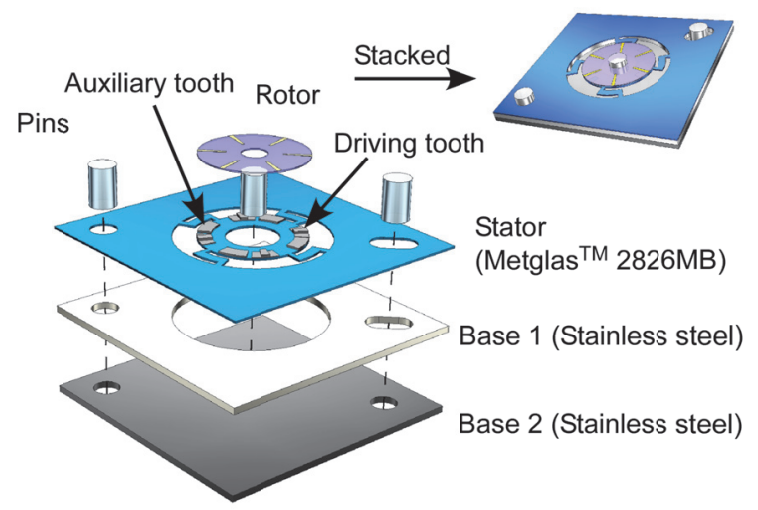

Figure 2: Schematic of a stacked prototype wireless resonant magnetoelastic miniature rotary motor.

Solid-State Sensors, Actuators, and Microsystems Workshop Hilton Head Island, South Carolina, June 3-7, 2012 
An on board coil and permanent magnets can be implemented to provide the driving $\mathrm{AC}$ and $\mathrm{DC}$ magnetic fields. For example, inductive coils for generating AC magnetic fields can be patterned on an underlying silicon substrate using standard micromachining techniques. On the same substrate, permanent magnet materials such as permalloy or samarium cobalt can be deposited and used to provide the DC fields required to bias the magnetoelastic material. However, for the prototype motor presented in this paper, external coils are used.

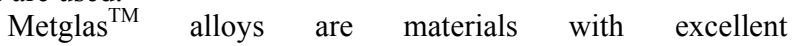
magnetostrictive properties as well as excellent mechanical properties. In this design, Metglas ${ }^{\mathrm{TM}} 2826 \mathrm{MB}$, an amorphous $\mathrm{NiFeMoB}$ alloy, is used. Its saturation magnetostriction is $12 \mathrm{ppm}$ and its DC permeability is larger than 50000 [9]. These materials are readily available in foils ( $\sim 28 \mu \mathrm{m}$ thick) and are easy to pattern utilizing PCM. Relative to other Metglas ${ }^{\mathrm{TM}}$ alloys and magnetoelastic materials, $2826 \mathrm{MB}$ also requires a small DC bias (about 2.5-3 Oe) and can resonate with a relatively small alternating field.

For a stator fabricated solely from thin Metglas ${ }^{\mathrm{TM}}$, careful consideration is required for the placement of the driving teeth. The number of driving teeth is usually the same as the number of antinodes in the desired mode shape for a resonant rotary motor [7]. The driving teeth are typically offset from the location of the antinodes in order to ensure elliptical motion of the tooth tip. However, because the Metglas ${ }^{\mathrm{TM}}$ stator is thin and light compared to any added driving teeth, the mass loading added by the driving teeth causes the antinodes to shift to the same location as the teeth. Consequently, the teeth no longer move elliptically and no rotation is generated. Therefore, as shown in Fig. 2 and 3, a design employing 8 teeth is proposed to address the issue. There are still four sets of teeth, but one set of teeth is a combination of two teeth: an auxiliary tooth and a driving tooth, each separated slightly from each other. The auxiliary teeth and driving teeth have the same mass so that the mass center and antinodes of the vibratory mode shape will be in the center between the two different teeth. However, the driving tooth is taller so only it will contact the rotor. As a result, the driving teeth can move in an elliptical manner, and drive the rotor tangentially.

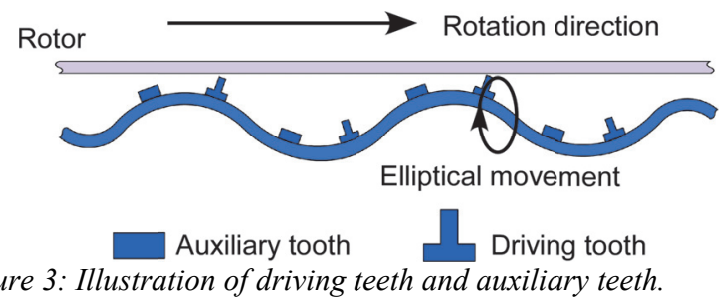

A custom magneto-mechanical harmonic finite element technique [10] is used to estimate modal displacements, shapes, and frequencies for the magnetoelastic material. Although magnetoelastic materials are generally non-linear, it is appropriate to use linearized constitutive equations describing the coupling between flux, field strength, stress, and strain in a magnetostrictive material:

$$
\begin{aligned}
& \vec{\sigma}=[C] \vec{\varepsilon}-\frac{[C][d]^{T}}{\mu_{o} \mu_{r}} \vec{B} \\
& \vec{H}=-\frac{[d][C]}{\mu_{o} \mu_{r}} \vec{\varepsilon}+\frac{1}{\mu_{0} \mu_{r}} \vec{B}
\end{aligned}
$$

where $\sigma$ is the stress vector, $C$ is the stiffness matrix, $\varepsilon$ is the strain, $d$ is the magnetostrictivity matrix, $B$ is the magnetic flux density vector, $H$ is the field strength vector, $\mu_{0}$ is the permeability of free space, and $\mu_{r}$ is the relative permeability. Equations (1) and (2) are implemented in this work utilizing COMSOL Multiphysics and coupled time-harmonic induction current and stress-strain frequency response modes. A detailed look at an FEA implementation for magnetostrictive materials is in [11]; the approach used in this work is modified for application to resonant actuators. The FEA results shown in Fig. 4 predict that a stator (inner diameter of $4 \mathrm{~mm}$ and outer diameter of $8 \mathrm{~mm}$ ) has the desired mode shape at a resonant frequency of about $11.43 \mathrm{kHz}$, with $100 \mathrm{~nm}$ out-of-plane deformation under harmonic excitation with amplitude of 2 Oe. Due to the orientation and size of the teeth and spring suspension, slightly different performance is predicted for different directions of alternating magnetic excitation. Simulation results suggest that applying magnetic field between the suspension springs gives the largest out-of-plane displacement amplitude. As desired, the vibration mode shape demonstrates that the antinodes are located in the center between a driving tooth and an auxiliary tooth.

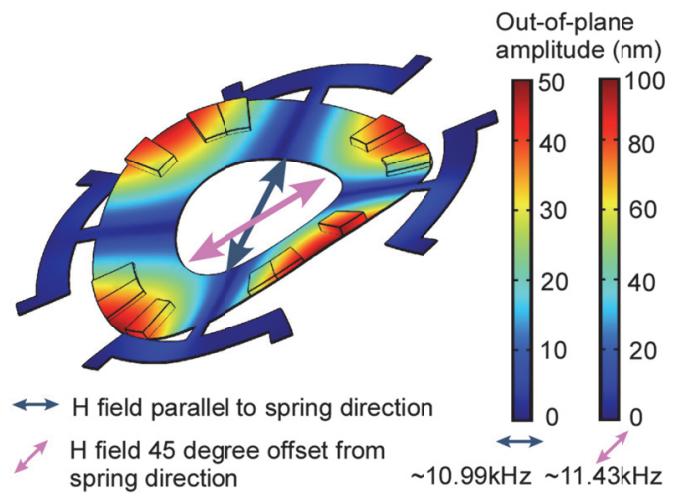

Figure 4: FEM results of out-of-plane stator vibration amplitude with different directions of AC magnetic field excitation.

\section{FABRICATION}

The fabrication process flow of a prototype motor is illustrated in Fig. 5. First, the magnetoelastic stator is batchpatterned using photochemical machining (PCM) [12] from the Metglas $^{\mathrm{TM}} 2826 \mathrm{MB}$ foil. The ring-shaped stator is patterned with inner diameter of $4 \mathrm{~mm}$ and outer diameter of $8 \mathrm{~mm}$. Other base layers are also fabricated using PCM from $0.5 \mathrm{~mm}$ thick stainless steel foils. The layers are stacked and aligned with pins, and bonded to each other with epoxy. The stainless steel auxiliary teeth and driving teeth are manually placed on the stator and fixed with epoxy. The rotor is electro-discharge-machined from magnetoelastic foil; it has a total mass of about $9 \mathrm{mg}$. A hub with diameter of about $2 \mathrm{~mm}$ is used to constrain the rotor. The overall size of the device is $2 \mathrm{~cm} \times 2 \mathrm{~cm}$ (Fig. 5).

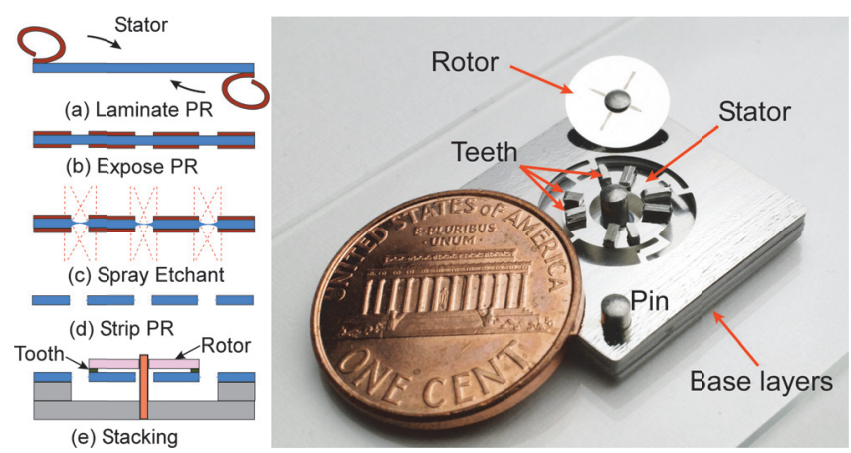

Figure 5: (Left) Fabrication process flow of a prototype magnetoelastic resonant rotary motor. (Right) Photograph of assembled prototype magnetoelastic resonant rotary motor. 


\section{EXPERIMENTAL METHODS AND RESULTS}

In the preliminary experiments reported in this paper, the motor is actuated wirelessly using two sets of external coils. Two large coils, shown in Fig. 6, are connected to a DC power supply with 2 A current, which corresponds to 3 Oe DC magnetic field. This is used to bias the magnetoelastic material into an operating region in which the strain is most sensitive to magnetic field. Another two small coils are placed inside the DC coils; these smaller coils provide an alternating magnetic field with amplitude of 2 Oe. The device under test (D.U.T.) is placed between the coils.

To measure the frequency response of the stator (before teeth have been added), a laser vibrometer and a network analyzer are used in conjunction (Fig. 6). The vibration mode shape can be determined by measuring a number of points along the rim of the stator; antinodes of a given mode shape exist at locations of strong response at a given frequency. The desired mode shape with four antinodes is confirmed to exist at $11.35 \mathrm{kHz}$, with $0.2 \mu \mathrm{m}$ unloaded out-of-plane deflection at the antinodes. As shown in Fig. 7, the frequency response is stable over at least 5 minutes of operation time.

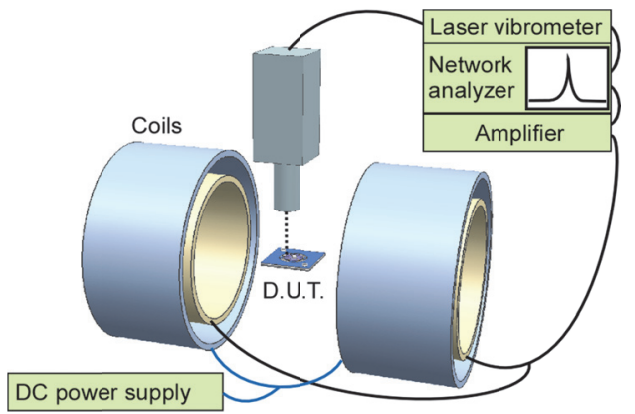

Figure 6: Experimental set up for stator vibration tests.

A video camera is used to record the rotor from above, and the video is analyzed frame-by-frame to derive the rotation speed and initial acceleration. In preliminary tests, a rotation rate of approximately $44 \mathrm{rpm}$ (4.6 radians/s) is obtained (Fig. 8). The angular resolution of about 23 milli-degree is calculated from the measured angular velocity and resonant frequency. The angular velocities from different trials are calculated from the recordings and are shown in Fig. 9. The angular acceleration, $\alpha$, before the angular velocity plateaus is derived from the data and the associated stall torque of $\sim 2 \mathrm{nN} \cdot \mathrm{m}$ is calculated using $\alpha$ and the moment of inertia of the rotor.

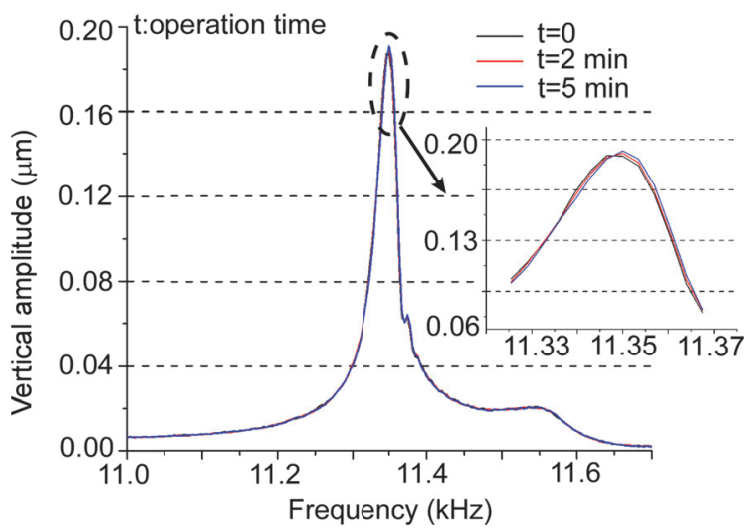

Figure 7: Frequency response of magnetoelastic stator under harmonic magnetic excitation.
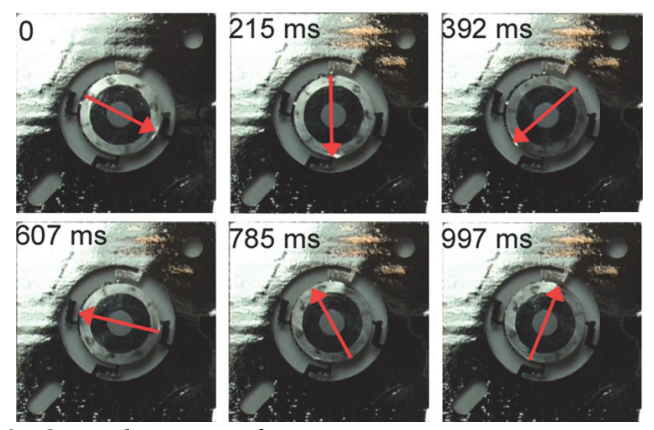

Figure 8: Optical images of rotor rotating

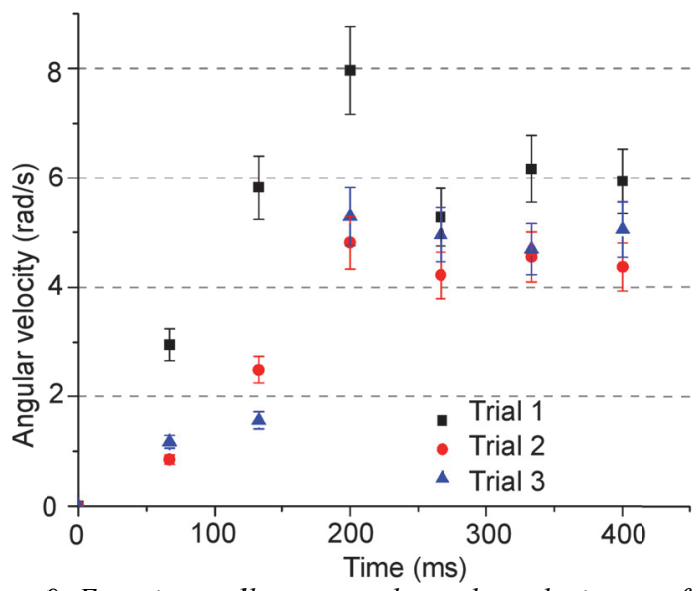

Figure 9: Experimentally measured angular velocity as a function of time under the excitation of a 3 Oe DC and a 2 Oe amplitude AC magnetic field at frequency of $11.35 \mathrm{kHz}$.

\section{DISCUSSION}

The prototype magnetoelastic wireless resonant motor demonstrates that the magnetoleastic material has significant potential for actuation in miniature and micro-scale devices. The main advantage - the wireless aspect of the actuation approach allows miniaturization and eliminates lead transfer actuator. Another obvious advantage is the ability to move large payloads; this non-optimized prototype carried a payload of about $9 \mathrm{mg}$.

A so-called silicon-Metglas ${ }^{\mathrm{TM}}$ stator is proposed and briefly analyzed. This design employs a silicon stator driven by a bonded magnetoelastic layer. Based on simulation results and the experimentally measured performance of stator prototypes, simulation parameters are derived and used in predicting performance (rotation speed and positioning resolution) for a silicon-Metglas ${ }^{\mathrm{TM}}$ architecture, as shown in Fig. 10. Simulations suggest that rotation speed is a function of the thickness of the silicon component, as shown in Fig. 11. By increasing the thickness of the silicon layer, the analyses indicate that the stepsize resolution and rotational speed can reach \pm 10 milli-degree and $1000 \%$ respectively. The predicted performance indicates improvement on rotation speed and resolution, which is crucial to many applications. The predicted performance of siliconMetglas $^{\mathrm{TM}}$ stator is compared with the measured performance of the prototype Metglas ${ }^{\mathrm{TM}}$ stator in Table I. The silicon-Metglas ${ }^{\mathrm{TM}}$ motor is expected to have a reduction in power consumption by decreasing the motor size. 


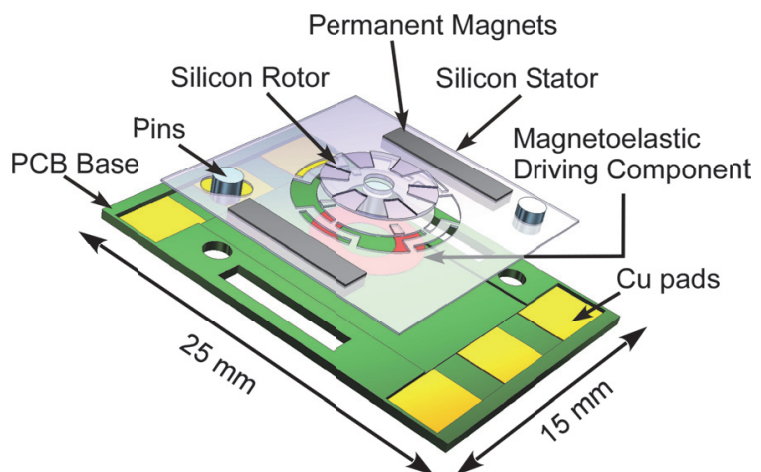

Figure 10: 3D Architecture of silicon-Metglas ${ }^{T M}$ rotary micromotor driven by $P C B$ circuits and permanent magnets.

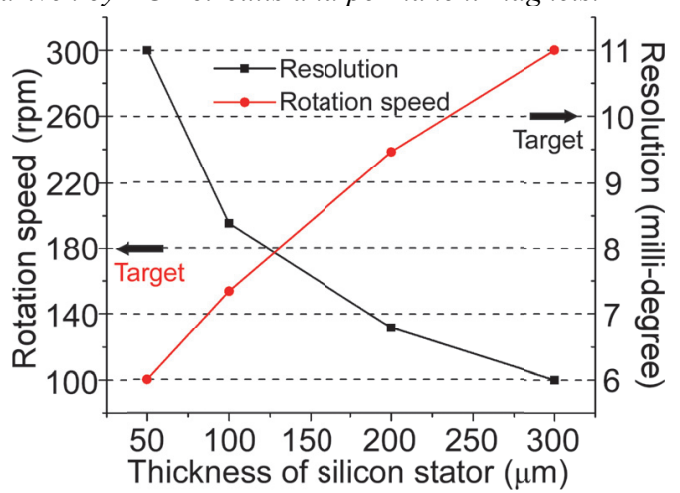

Fig. 11: Simulated dependence of step-size resolution and rotation speed on thickness of silicon stator.

Table I: Experimental performance of prototype magnetoelastic rotary motor and estimated target performance of siliconMetglas ${ }^{T M}$ stator architecture. (S) denotes simulated results while other results are measured.

\begin{tabular}{|l|c|c|}
\hline Performance & $\begin{array}{c}\text { Prototype } \\
\text { (Measured) }\end{array}$ & $\begin{array}{c}\text { Si-Metglas } \\
\text { (Predicted) }\end{array}$ \\
\hline Rotation speed $(\mathrm{rpm})$ & $\sim 44$ & $180(\mathrm{~S})$ \\
\hline Stall torque $(\mathrm{nN} \cdot \mathrm{m})$ & $\sim 2$ & - \\
\hline Step size $($ milli-degree) & $\sim 23$ & $10(\mathrm{~S})$ \\
\hline Input Power & $84 \mu \mathrm{W}(\mathrm{S})$ & $74 \mu \mathrm{W}(\mathrm{S})$ \\
\hline Output Power & $\sim 9 \mathrm{nW}$ & - \\
\hline
\end{tabular}

\section{CONCLUSION}

This work presents the analysis, fabrication, and experimental results for magnetoelastically-actuated, chip-scale rotary motors. The prototype design is actuated wirelessly by lithographically micromachined magnetoelastic foil. The Metglas ${ }^{\mathrm{TM}}$ stator has diameter of $8 \mathrm{~mm}$. A new approach that employs auxiliary teeth and driving teeth is used to customize the resonant behavior of the stator. In preliminary tests, external coils are used to provide a 3 Oe DC and a 2 Oe amplitude AC magnetic field. A rotation speed of about $44 \mathrm{rpm}$ is achieved. Associated stall torque and output power are calculated to be $\sim 2 \mathrm{nN} \cdot \mathrm{m}$ and $\sim 9 \mathrm{nW}$, respectively. The angular step size is about 23 milli-degree.

As the next step, a silicon-Metglas ${ }^{\mathrm{TM}}$ motor is proposed and analyzed using parameters derived from simulation results and experimental prototype results. Predicted results suggest significant performance improvement in rotational speed, angular resolution and power consumption. The results and analysis in this work indicate that bulk magnetoelastic material is a viable candidate for wireless actuation in MEMS devices. Future work will focus on the proposed silicon-Metglas ${ }^{\mathrm{TM}}$ motor.

\section{ACKNOWLEDGEMENTS}

The authors acknowledge Metglas, Inc. for the samples provided for this project. This work was supported in part by a DARPA project (PASCAL), and the University of Michigan.

\section{REFERENCES}

[1] L.S. Fan, Y.C. Tai, R.S. Muller, "IC-processed Electrostatic Micromotors," Sensors and Actuators, 20, (1989), pp 41-47.

[2] V. Kaajakari, A. Lal, "Micromachined ultrasonic motor based on parametric polycrystalline silicon plate excitation," Sensors and Actuators A, 137, (2007), pp. 120-128.

[3] C.H. Ahn, Y.J. Kim, M.G. Allen, "A Planar Variable Reluctance Magnetic Micromotor with Fully Integrated Stator and Coils," Journal of Microelectromechanical Systems, 2 (4), (1993), pp. 165-173.

[4] J.S. Park, L.L. Chu, A.D. Oliver, Y.B. Gianchandani, "BentBeam Electrothermal Actuators - Part II: Linear and Rotary Microengines," Journal of Microelectromechanical Systems, 10 (2), (2001), pp. 255-262.

[5] C.A. Grimes, S.C. Roy, S. Rani, and Q.Y. Cai, "Theory, Instrumentation and Applications of Magnetoelastic Resonance Sensors: A Review," Sensors, 11, (2011), pp. 2809-2844.

[6] M. Suzuki, K. Tani, T. Sakahura, "Development of a new type piezoelectric micromotor," Sensors and Actuators A, 83, (2000), pp. 244-248.

[7] A. Iino, K. Suzuki, M. Kasuga, M. Suzuki, T. Yamanaka, "Development of a self-oscillating ultrasonic micro-motor and its application to a watch," Ultrasonics, 38, (2000), pp. 54-59.

[8] Ph. Robert, J.S. Danel, P. Villard, "The electrostatic ultrasonic micromotor," Journal of Micromechanics and Microengineering, 7, (1997), pp. 170-172.

[9] Metglas, Inc. "Magnetic Alloy 2826MB (nickel-based) Technical Bulletin". [Online]. Available: http://www.metglas.com

[10] S.R. Green, Y.B. Gianchandani, "Wireless Magnetoelastic Monitoring of Biliary Stents," Journal of Microelectromechanical Systems, 18 (1), (2009), pp. 64-78.

[11] J. Benatar, "FEM Implementations of Magnetostrictive-Based Applications", MS thesis, Univ. of Maryland, 2005.

[12] ASM Handbook, 16, ASM International, 1989.

\section{CONTACT}

*J. Tang tel: +1-765-714-5838; juntang@umich.edu 